PREPARED FOR THE U.S. DEPARTMENT OF ENERGY, UNDER CONTRACT DE-AC02-76CH03073

PPPL-3768

PPPL-3768

UC-70

First Evidence for the Existence of Odd Toroidal Alfvén Eigenmodes (TAEs) from the Simultaneous Observation of Even and Odd TAEs on the Joint European Torus

by

G.J. Kramer, S.E. Sharapov, R. Nazikian, N.N. Gorelenkov, R. Budny, and contributors to the JET-EFDA Workprogramme

January 2003
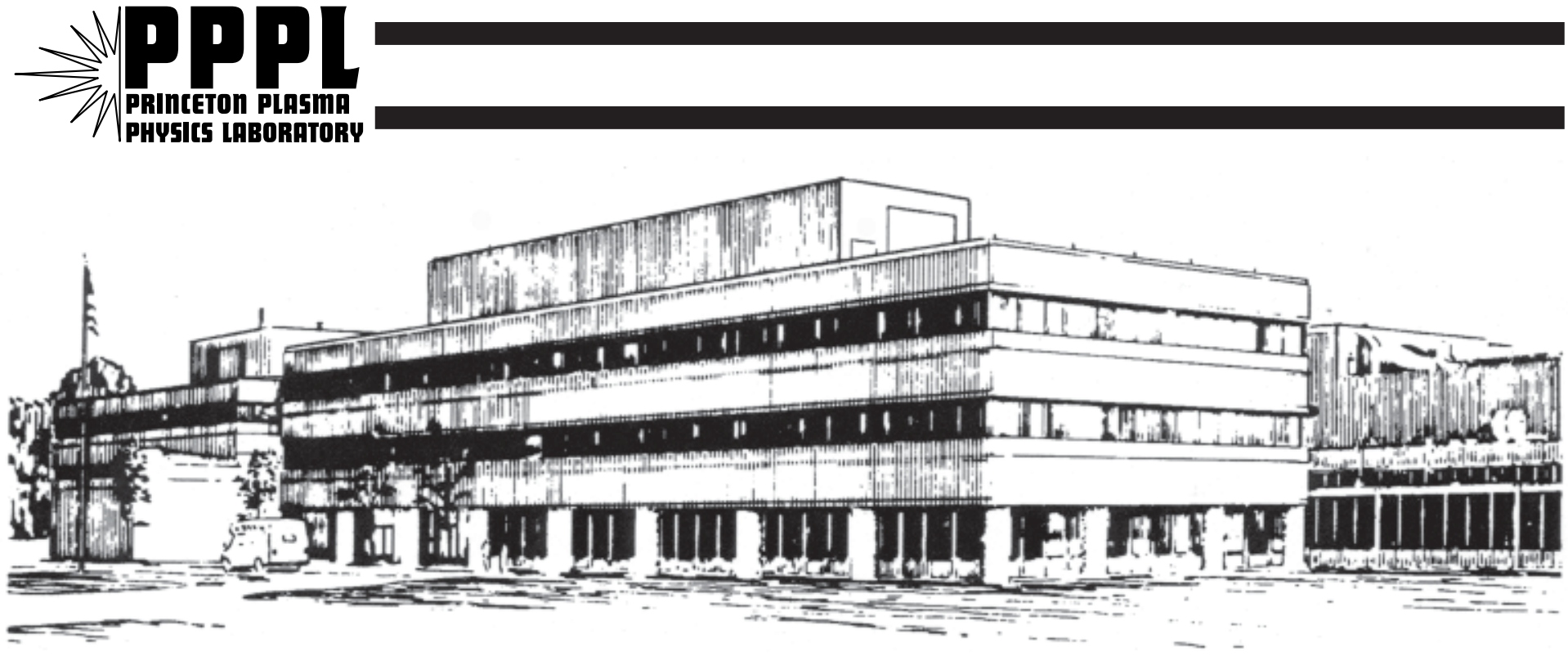

PRINCETON PLASMA PHYSICS LABORATORY PRINCETON UNIVERSITY, PRINCETON, NEW JERSEY 


\section{PPPL Reports Disclaimer}

This report was prepared as an account of work sponsored by an agency of the United States Government. Neither the United States Government nor any agency thereof, nor any of their employees, makes any warranty, express or implied, or assumes any legal liability or responsibility for the accuracy, completeness, or usefulness of any information, apparatus, product, or process disclosed, or represents that its use would not infringe privately owned rights. Reference herein to any specific commercial product, process, or service by trade name, trademark, manufacturer, or otherwise, does not necessarily constitute or imply its endorsement, recommendation, or favoring by the United States Government or any agency thereof. The views and opinions of authors expressed herein do not necessarily state or reflect those of the United States Government or any agency thereof.

\section{Availability}

This report is posted on the U.S. Department of Energy's Princeton Plasma Physics Laboratory Publications and Reports web site in Fiscal Year 2003. The home page for PPPL Reports and Publications is: http://www.pppl.gov/pub_report/

DOE and DOE Contractors can obtain copies of this report from:

U.S. Department of Energy

Office of Scientific and Technical Information

DOE Technical Information Services (DTIS)

P.O. Box 62

Oak Ridge, TN 37831

Telephone: (865) 576-8401

Fax: (865) 576-5728

Email: reports@adonis.osti.gov

This report is available to the general public from:

National Technical Information Service

U.S. Department of Commerce

5285 Port Royal Road

Springfield, VA 22161

Telephone: $1-800-553-6847$ or

(703) $605-6000$

Fax: (703) 321-8547

Internet: http://www.ntis.gov/ordering.htm 


\title{
First evidence for the existence of odd Toroidal Alfvén Eigenmodes (TAEs) from the simultaneous observation of even and odd TAEs on the Joint European Torus.
}

\author{
G.J. Kramer ${ }^{1}{ }^{*}$ S.E. Sharapov ${ }^{2}$, R. Nazikian ${ }^{1}$, \\ N.N. Gorelenkov ${ }^{1}$, R. Budny ${ }^{1}$, and JET-EFDA contributors ${ }^{\dagger}$ \\ ${ }^{1}$ Princeton Plasma Physics Laboratories, \\ P.O.box 451, Princeton, New Jersey 08543 and \\ ${ }^{2}$ Euratom/UKAEA Fusion Association, \\ Culham Science centre, Abingdon OX14 3DB, U.K.
}

(Dated: January 14, 2003)

\begin{abstract}
Experimental evidence is presented for the existence of the theoretically predicted odd Torodicity induced Alfvén Eigenmode (TAE) from the simultaneous appearence of odd and even TAEs in a normal shear discharge. The modes are observed in low central magnetic shear plasmas created by injecting lower hybrid current drive. A fast ion population was created by applying ion cyclotron heating at the high field side to excite the TAEs in the weak magnetic shear region. The odd TAEs were identified from their frequency, mode number, and timing relative to the even TAEs.
\end{abstract}

$\lceil$ See annex of J. Pamela et al., "Overview of Recent JET Results" (Proc. 19th Int. IAEA Conf., Lyon 2002) 


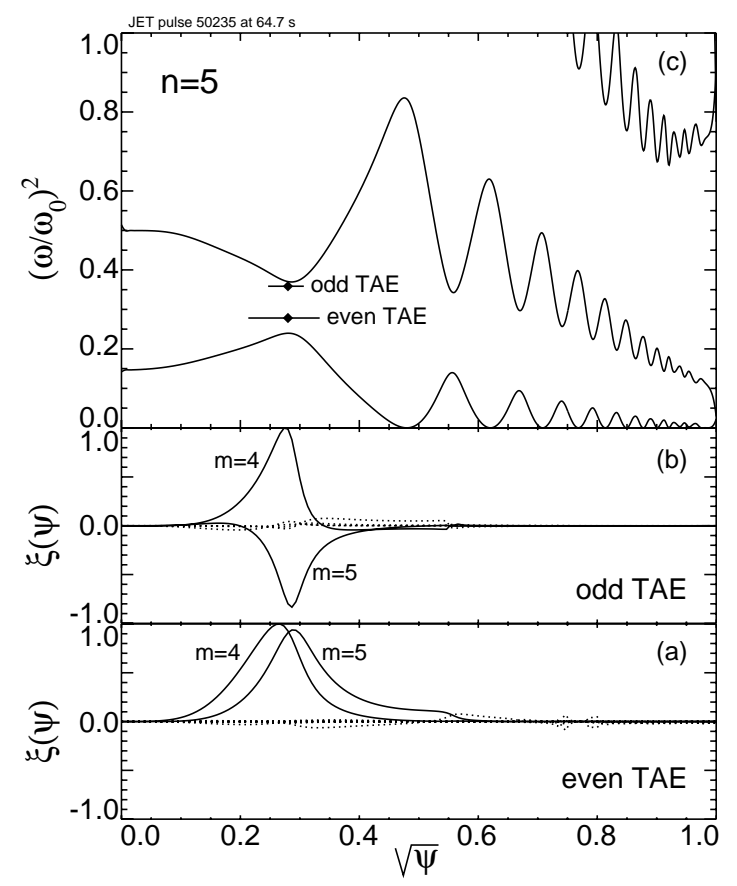

FIG. 1: NOVA-K solution for an $n=5$ even (a) and odd (b) TAE and their locations in the Alfvén continuum gap (c) normalized to $\omega_{0}=B / R \sqrt{\rho(0)}$ with $\rho(0)$ the central mass density. The modes are located at $\epsilon=0.1$, with $s=0.272$, and $\alpha=0.131$. The dotted curves in (a) and (b) are poloidal components other than $\mathrm{m}=4$ and 5 .

At the time of the theoretical discovery of Torodicity induced Alfvén Eigenmodes (TAE) in the low magnetic shear region of fusion plasmas, predictions were made for two modes with the same toroidal mode number, $n$, in the TAE gap [1-4]. The two modes are formed by the coupling of two poloidal harmonics, $m$ and $m+1$. The even mode, which resides at the bottom end of the TAE gap, is formed by the coupling of the poloidal harmonics with the same sign whereas the odd mode, which resides at the top end of the TAE gap, has opposite signs between its two poloidal components as can be seen in figure 1. Because of this difference the even TAE has a ballooning mode structure and the odd TAE has an antiballooning structure. These two TAEs can exist when the following condition is fulfilled: $s^{2}<\epsilon<s$ (with $s=r / q \mathrm{~d} q / \mathrm{d} r$ the magnetic shear, $\epsilon=r / R$ the inverse aspect ratio and $R$ the tokamak major radius) [3]. When $\epsilon>s$ a spectrum of multiple TAEs differing in the number of radial nodes was found to exist in one gap [5]. The low shear TAEs can only exist when the normalized pressure gradient, $\alpha=-2\left(R q^{2} / B^{2}\right) p^{\prime}$ (with $q$ the magnetic safety factor, $B$ the magnetic field strength, and $p^{\prime}=\mathrm{d} p / \mathrm{d} r$ the radial derivative of the pressure) 


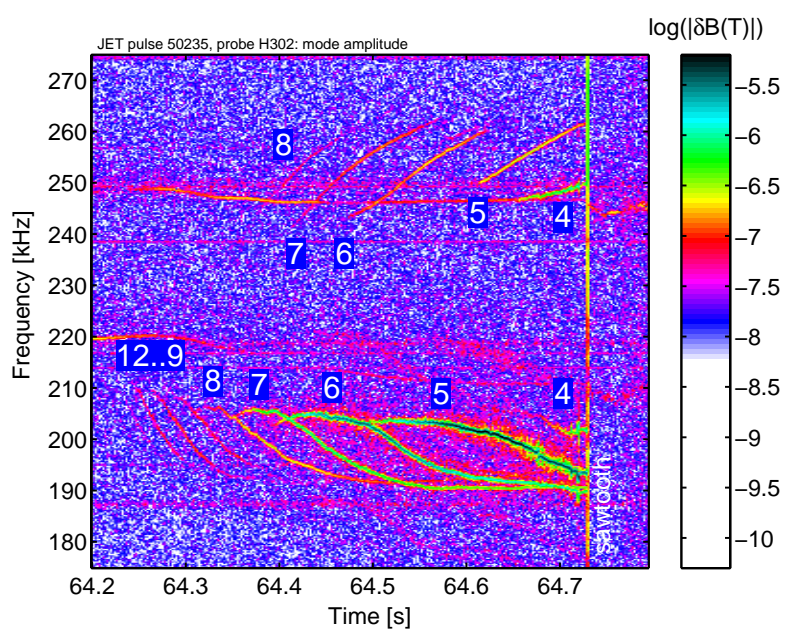

FIG. 2: Spectrogram of the even $(f=190-210 \mathrm{kHz})$ and odd $(f=250-265 \mathrm{kHz})$ TAEs labeled with respective toroidal mode numbers.

is lower than a critical value. For the even mode this critical value is given by $\alpha_{c}^{E} \approx 3 \epsilon+2 s^{2}$ and for the odd mode $\alpha_{c}^{O} \approx 3 \epsilon-2 s^{2}$ [2]. The odd mode only exists because of finite aspect ratio effects and its critical $\alpha$ is lower than the one for the even mode which indicates that the odd mode ceases to exist before the even mode disappears if the central pressure gradient increases. This suggests that the even mode is more robust than the odd mode.

Experimentally so far, only the even mode has been observed unambiguously in large Tokamaks [6-8], usually during sawtooth stabilization experiments with Ion Cyclotron range of frequency Heating (ICRH). In those ICRH experiments an extended region of low shear is formed up to half of the plasma minor radius where the even TAEs reside. Up till now there has been no unambiguous identification of the odd TAE in tokamak experiments despite the early prediction of its existence.

In this letter we present unambiguous experimental evidence for the existence of the odd TAEs from the co-existence of even and odd TAEs in a JET discharge. We further address the question why the odd TAEs are observed so rarely in tokamaks.

In fig. 2 a spectrogram of the magnetic fluctuations as measured with a Mirnov coil at the plasma outer midplane is shown where a number of modes with $n$ deceasing in time from 12 to 4, show up between 190 and $210 \mathrm{kHz}$. These modes chirp down in frequency. Another set of modes with $n$ deceasing in time from 8 to 4 and chirping up in frequency when time progresses is visible between 240 and $265 \mathrm{kHz}$. At the giant sawtooth crash of 


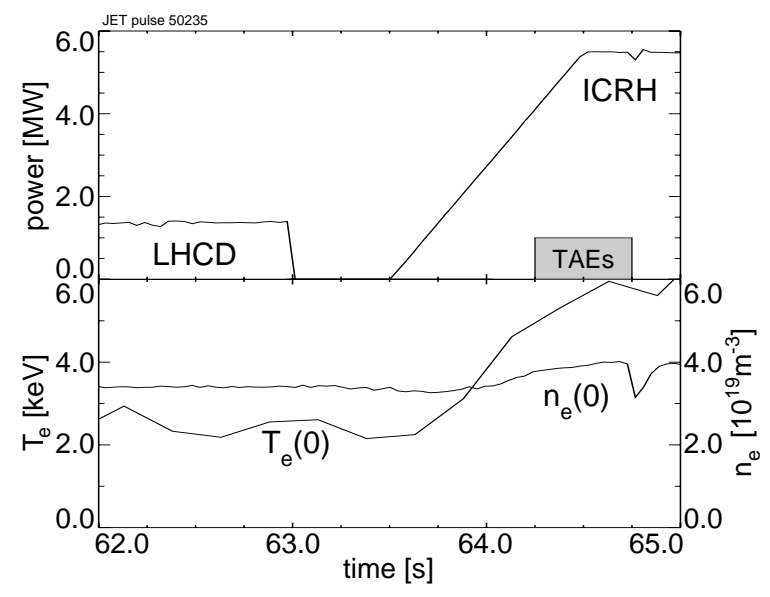

FIG. 3: (a) ICRH and LHCD heating wave forms and (b) central electron density and temperature.

$64.75 \mathrm{~s}$ these modes disappear. The plasma current in this discharge (JET pulse 50235) was 2.5 MA, the toroidal magnetic field at the magnetic axis was $2.6 \mathrm{~T}$. The heating wave forms of ICRH and Lower Hybrid Current Drive (LHCD, applied from 59 to $63 \mathrm{~s}$ ) are shown in fig. 3 together with the on axis electron density on axis and temperature. The first harmonic hydrogen ICRH resonance was at $2.81 \mathrm{~m}$ which is $0.15 \mathrm{~m}$ to the high field side from the plasma center. No neutral beams were injected in this discharge but from the appearance of the the even TAEs the evolution of the central $q$-profile can be deduced [9]. The Alfvén frequency at the $q=1$ surface before the sawtooth crash at $64.75 \mathrm{~s}$ is $240 \mathrm{kHz}$, estimated from $\omega_{T A E}=B /\left(\sqrt{\mu_{0} \rho} 2 R_{0} q\right)$ ( $\rho$ the mass density). A thorough comparison between measured and simulated frequencies is difficult because the plasma rotation, which induces a Doppler shift, was not measured. The rotation, however, was estimated to be around $1 \mathrm{kHz}$ (from a line integrated nickel impurity measurement).

The pattern of the modes around $200 \mathrm{kHz}$ is typical for even TAEs at the bottom of the TAE gap. The frequency chirping is caused by a slowly decreasing central magnetic safety factor, $q_{0}[10,11]$.

The CASTOR code [12] was used with experimentally determined plasma profiles reconstructed with EFIT to evaluate the frequency behavior of the TAEs as a function of $q_{0}$. It was found that the low shear region satisfied the condition: $s^{2}<\epsilon<s$, so that only two TAEs are expected to exist [3]. Furthermore, the value of $\alpha=0.131$ is just (well) below the critical value for the odd (even) TAEs. The relevant model for this discharge is thus the one with two TAEs only [3]. As soon as the threshold $q_{0}$ is passed for exciting the TAEs 


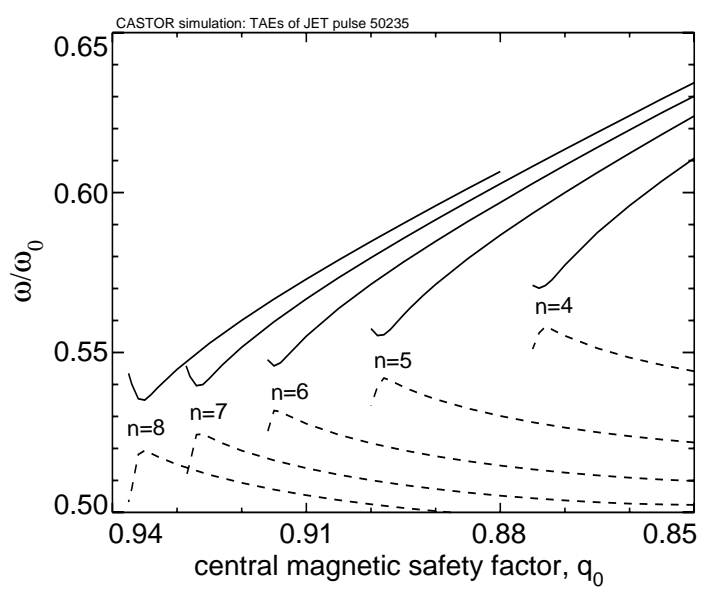

FIG. 4: CASTOR simulation of the frequency behavior of the observed TAEs as a function of (decreasing) $q_{0}$. Solid curves: odd TAEs, dashed curves: even TAEs, and $\omega_{0}$ as in fig. 1.

with mode number $n$, the even and odd mode are both found from the code (fig. 4). The even TAEs show a small frequency up-chirp followed by a steady down-chirp that eventually comes to a halt. The odd TAEs show a small frequency down-chirp followed by a steady up-chirp that does not show signs of frequency saturation as the even modes do.

The different frequency behavior of the odd and even TAEs is explained as follows. When $q_{0}$ decreases, the even TAEs follow the bottom of the TAE gap to a region where they can couple with global TAEs that reside outside the $q=1$ surface thereby becoming far less sensitive to variations in $q_{0}$. The change from a core localized to global mode structure is revealed form CASTOR calculations. The odd TAEs follow the top of the TAE gap when $q_{0}$ decreases and they eventually disappear in the upper Alfvén continuum without coupling to global modes [4].

The simulated frequency behavior of the even and odd TAEs compare very well with the modes observed experimentally at around 200 and $250 \mathrm{kHz}$. The observed modes are excited sequentially with the highest $n$ first as expected for a decreasing $q_{0}$. The frequency of the modes around $200 \mathrm{kHz}$ show a slight up-chirp when they appear followed by a down-chirp that comes to a stop after a while similar to the even TAEs in the simulations. The modes at around $250 \mathrm{kHz}$ in the experiment chirp up monotonically and they show no sign of a decreasing frequency chirp before they disappear which matches well with the frequency behavior of the odd TAEs as found in the CASTOR simulations.

For the modes to be excited in experiments the fast particle drive has to overcome the 
TABLE I: Damping rates from NOVA-K for odd (top) and even (bottom) TAEs at $q_{0}=0.87$.

\begin{tabular}{rcccc}
\hline \hline & \multicolumn{3}{c}{ odd TAEs } & \\
n & 5 & 6 & 7 & 8 \\
ion Landau [\%] & -0.008 & -0.004 & -0.002 & -0.002 \\
electron Landau [\%] & -0.031 & -0.028 & -0.037 & -0.025 \\
collisional [\%] & -0.016 & -0.090 & -0.368 & -0.601 \\
radiative [\%] & -0.001 & -0.010 & -0.017 & -0.019 \\
total [\%] & -0.056 & -0.132 & -0.424 & -0.647 \\
\hline $\mathrm{n}$ & & even TAEs & & 8 \\
ion Landau [\%] & 5 & -0.016 & 7 & -0.005 \\
electron Landau [\%] & -0.041 & -0.004 & -0.007 & -0.014 \\
collisional [\%] & -0.048 & -0.196 & -0.016 & -4.387 \\
radiative [\%] & -0.273 & -2.059 & -0.373 & -2.704 \\
total [\%] & -1.286 & -2.275 & -2.395 & -7.110 \\
\hline \hline
\end{tabular}

damping of the modes. We have used the NOVA-K code [13] to study the various damping mechanisms and the fast particle drive mechanism to investigate if the drive is sufficient for the excitation of the even and odd modes. In a TAE study at JT-60u it was reported that the critical fast particle $\beta$ for the odd TAEs is more than ten times the one for the even TAEs [14] and because of that the odd TAEs were not observed there.

We have calculated the damping rates for the odd and even TAEs with $n$ between 5 and 8. The profiles that were needed for NOVA-K were obtained from TRANSP [15] at $64.5 \mathrm{~s}$. At that time $q_{0}$ computed by TRANSP was 0.87 . From the results for the damping rates as shown in table I it can be seen that the total damping rate for both the odd and even modes decreases with decreasing $n$. The main damping mechanism for most of the odd modes is the collisional damping [16]. Only for the $n=5$ mode the electron Landau damping is the strongest. Except for the $n=8$ mode where the collisional damping is the strongest, the radiative damping is the main damping mechanism for the even modes due to its frequency being close to the lower continuum. (The radiative damping was calculated along the lines of refs. $[4,17])$. All the modes studied here were located well inside the TAE gap so that they 


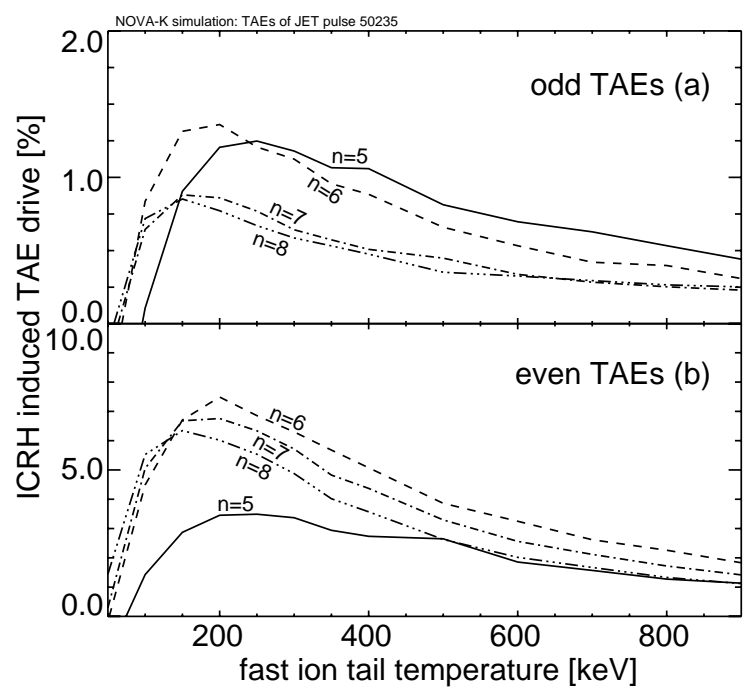

FIG. 5: NOVA-K simulation the the ICRH induced fast particle drive for (a) the odd TAEs and (b) the even TAEs as a function of the fast ion tail temperature.

did not interact with the Alfvén continuum, hence the continuum damping was negiglible.

The ICRH induced fast particle drive was also calculated with NOVA-K for the even and odd modes with $n$ between 5 and 8 as a function of the hot ion tail temperature (fig. 5). In the calculations for the fast ion drive, finite orbit width and finite Lamor radius effects [1820] were included. The maximum drive for the even TAEs was calculated to be between 3.0 and $7.5 \%$ which is sufficient to excite the modes with $n$ between 5 and 7 . The maximum drive for the odd TAEs, between 0.8 to $1.4 \%$ depending on $n$, is reached for tail temperatures less than $250 \mathrm{keV}$ and is sufficient to overcome the damping (table I). In this experiment the ICRH resonance layer was located $15 \mathrm{~cm}$ away from the plasma center toward the high field side. This helped to increase the fast ion drive for the odd TAEs significantly compared to on-axis ICRH. For on-axis ICRH the threshold fast ion tail temperature for exciting the odd TAEs shifted to higher tail temperatures, 100 to $200 \mathrm{keV}$ depending on $n$. The maximum odd TAE drive for on-axis heating was reached at much higher tail temperatures, between 200 and $400 \mathrm{keV}$ and the maximum drive was found to be more than three times less than for the high field side heating, insufficient to excite the odd TAEs.

The large difference in the drive between the odd and even TAEs can be understood from the fact that the even TAE has a ballooning structure (localized at the low field side) whereas the odd TAE has an anti-ballooning structure (localized at the high field side). The 
TAEs are excited by the trapped fast particles so it is much more difficult to excite the odd TAE than the even TAE. Shifting the resonance layer to the high field side increased the interaction between the fast particles and the odd TAE to a level where the odd modes were excited.

The ICRH induced fast ion tail temperature was estimated to be in the range of 200 to $400 \mathrm{keV}$ from the TRANSP calculations so the fast particle drive as calculated with NOVA-K was sufficient to destabilize both the even and the odd TAEs in this discharge.

The mode amplitude saturation is related to the ratio of the drive and damping rates. The TAE amplitude saturation is directly related to the quotient of the linear TAE growth rate, $\gamma_{L}$, in our case from the accelerated hydrogen minority ions, and the damping rate, $\gamma_{d}$. Close to the threshold the non linear mode saturation is proportional to $\sqrt{\gamma_{L} / \gamma_{d}-1}$ and well above the threshold it is proportional to $\left(\gamma_{L} / \gamma_{d}\right)^{2 / 3}[21,22]$.

Experimentally, we observe that the modes with lower $n$ have a larger amplitude than the high $n$ modes. For the same $n$ we also observe that the even TAE (around $200 \mathrm{kHz}$ ) is excited more strongly than the odd TAE (around $250 \mathrm{kHz}$ ). These observations are in agreement with the NOVA-K simulations where it was found that high- $n$ TAEs have larger damping rates than low- $n$ TAEs. Moreover, The NOVA-K modeling of the ICRH drive showed that the odd TAEs have a much weaker drive than the even TAEs which is consistent with the observed mode saturation level.

From all of the above evidence we conclude that the modes around $200 \mathrm{kHz}$ are the even TAEs and the modes around $250 \mathrm{kHz}$ are the odd TAEs. The final question that remains is why the odd and the even TAEs appear together in this discharge whereas in all other discharges only the even TAEs are observed.

As pointed out in the introduction, two conditions have to be full filled for the existence of odd TAEs in Tokamaks: i) a low magnetic shear region in the core, and ii) a flat central pressure profile. Moreover, the ICRH accelerated fast ion population should supply enough drive to excite the the odd TAEs. These conditions were all met in this discharge.

When the TAEs were excited, the plasma inductance $\left(\ell_{i}\right)$ was 1.1 which is significantly lower than $\ell_{i}=1.2$ to 1.3 for discharges where only the even TAEs observed. This low $\ell_{i}$ indicates that the current distribution profile was broader and hence the $q$ profile was flatter than usual in this discharge. The LHCD that was injected before the ICRH was switched on, had reduced the central magnetic shear in such a way that both the odd and even TAEs 
can exist. The odd and even TAEs appeared during the ICRH ramp-up phase (fig. 3) when the fast ion pressure was building up. The ICRH resonance layer was located at the high field side of the plasma center. This increased the the drive for the odd TAEs to a level where the modes were excited.

In normal shear discharges with ICRH driven TAEs, the LHCD pulse before the application of ICRH is absent so the ICRH has to create the low central shear region first. During that time, the central pressure is able to become unfavorably peaked for odd TAEs. Moreover, the on-axis ICRH that is often used in experiments is highly unfavourable for the excitation of the odd TAEs. We can thus explain the successful observation of the odd TAEs in this discharge with the combination of LHCD before the ICRH and the high-field off-axis ICRH deposition.

For the first time the odd TAEs, which were predicted to exist in the core of large scale fusion plasmas, have been identified unambiguously by comparing experimental results with CASTOR and NOVA-K simulations. The odd TAEs were excited because of the creation of a central low magnetic shear region with LHCD. Moreover, the ICRH was deposited away from the plasma center at the high field side which enhanced the fast particle drive significantly for the odd TAEs.

This work has been conducted partly under the European Fusion Development Agreement and partly by DOE Contract No. DE-AC02-76-CH0373 and by Euratom and the UK Department of Trade and Industry.

* gkramer@pppl.gov

[1] S. Poedts et al. Plasma Phys. Control. Fusion 34, 1397 (1992).

[2] G.Y. Fu Phys. of Plasmas 2, 1029 (1995).

[3] H. Berk et al. Phys. of Plasmas 23401 (1995).

[4] G.Y. Fu et al. Phys. of Plasmas 3, 4036 (1996).

[5] J. Candy et al. Phys. Lett. A 215, 299 (1996).

[6] M. Saigusa et al. Plasma Phys. Control. Fusion 40, 1647 (1998).

[7] S. Bernabei et al. Phys. of Plasmas 6, 1880 (1999).

[8] S.E. Sharapov et al. Nucl. Fusion 39, 373 (1999). 
[9] G.J. Kramer et al. Plasma Phys. Control. Fusion 40, 863 (1998).

[10] M. Saigusa et al. Plasma Phys. Control. Fusion 37, 295 (1995).

[11] G.J. Kramer et al. Phys. Rev. Lett. 83, 2961 (1999).

[12] S. Poedts et al. J. Comput. Phys. 142, 165 (1993).

[13] C.Z. Cheng, Phys. Rep. 211, 1 (1992).

[14] G.J. Kramer et al. Nucl. Fusion 41, 1135 (2001).

[15] R.V. Budny, et al. Nucl. Fusion 35, 1497 (1995) and references therein.

[16] G.Y. Fu et al. Phys. of fluids B 5, 4040 (1993).

[17] H.L. Berk et al. Phys. of fluids B 4, 1806 (1992).

[18] H.L. Berk, et al. Phys. Lett. 162A, 475 (1992).

[19] B.N. Breizman and S.E. Sharapov, Plasma Phys. Control. Fusion 37, 1057 (1995).

[20] N.N. Gorelenkov, et al. Phys. of Plasmas 6, 2802 (1999).

[21] H.L. Berk et al. Phys. Rev. Lett. 76, 1256 (1996).

[22] N.N. Gorelenkov et al. Phys. of Plasmas 6, 629 (1999). 


\section{External Distribution}

Plasma Research Laboratory, Australian National University, Australia

Professor I.R. Jones, Flinders University, Australia

Professor João Canalle, Instituto de Fisica DEQ/IF - UERJ, Brazil

Mr. Gerson O. Ludwig, Instituto Nacional de Pesquisas, Brazil

Dr. P.H. Sakanaka, Instituto Fisica, Brazil

The Librarian, Culham Laboratory, England

Mrs. S.A. Hutchinson, JET Library, England

Professor M.N. Bussac, Ecole Polytechnique, France

Librarian, Max-Planck-Institut für Plasmaphysik, Germany

Jolan Moldvai, Reports Library, MTA KFKI-ATKI, Hungary

Dr. P. Kaw, Institute for Plasma Research, India

Ms. P.J. Pathak, Librarian, Insitute for Plasma Research, India

Ms. Clelia De Palo, Associazione EURATOM-ENEA, Italy

Dr. G. Grosso, Instituto di Fisica del Plasma, Italy

Librarian, Naka Fusion Research Establishment, JAERI, Japan

Library, Plasma Physics Laboratory, Kyoto University, Japan

Research Information Center, National Institute for Fusion Science, Japan

Dr. O. Mitarai, Kyushu Tokai University, Japan

Library, Academia Sinica, Institute of Plasma Physics, People's Republic of China

Shih-Tung Tsai, Institute of Physics, Chinese Academy of Sciences, People's Republic of China

Dr. S. Mirnov, TRINITI, Troitsk, Russian Federation, Russia

Dr. V.S. Strelkov, Kurchatov Institute, Russian Federation, Russia

Professor Peter Lukac, Katedra Fyziky Plazmy MFF UK, Mlynska dolina F-2, Komenskeho Univerzita, SK-842 15 Bratislava, Slovakia

Dr. G.S. Lee, Korea Basic Science Institute, South Korea

Institute for Plasma Research, University of Maryland, USA

Librarian, Fusion Energy Division, Oak Ridge National Laboratory, USA

Librarian, Institute of Fusion Studies, University of Texas, USA

Librarian, Magnetic Fusion Program, Lawrence Livermore National Laboratory, USA

Library, General Atomics, USA

Plasma Physics Group, Fusion Energy Research Program, University of California at San Diego, USA

Plasma Physics Library, Columbia University, USA

Alkesh Punjabi, Center for Fusion Research and Training, Hampton University, USA

Dr. W.M. Stacey, Fusion Research Center, Georgia Institute of Technology, USA

Dr. John Willis, U.S. Department of Energy, Office of Fusion Energy Sciences, USA

Mr. Paul H. Wright, Indianapolis, Indiana, USA 
The Princeton Plasma Physics Laboratory is operated by Princeton University under contract with the U.S. Department of Energy.

\author{
Information Services \\ Princeton Plasma Physics Laboratory \\ P.O. Box 451 \\ Princeton, NJ 08543
}

Phone: 609-243-2750

Fax: 609-243-2751

e-mail: pppl_info@pppl.gov

Internet Address: http://www.pppl.gov 